\title{
Interaction between two predictors of functional outcome after revascularization in ischemic cardiomyopathy: Left ventricular volume and amount of viable myocardium
}

\author{
Mohammad Hossein Mandegar, MD, Mohammad Ali Yousefnia, MD, Farideh Roshanali, MD, \\ Hussein Rayatzadeh, MD, and Farshid Alaeddini, MD, PhD
}

Objective: In patients with ischemic cardiomyopathy and substantial amounts of dysfunctional but viable myocardium, revascularization cannot always improve the left ventricular ejection fraction. We sought to investigate the interaction between the left ventricular volume and the amount of viable myocardium to predict the left ventricular ejection fraction increase after revascularization.

Methods: Eighty-five consecutive patients with a depressed left ventricular ejection fraction (mean: $27.3 \% \pm 5.2 \%$ ) underwent coronary artery bypass grafting after a dobutamine stress echocardiography had determined that they had at least 4 viable segments. Six months after coronary artery bypass grafting, left ventricular ejection fraction and regional wall motion were reassessed.

Results: Although the left ventricular ejection fraction was expected to recover more than $5 \%$ in all 85 patients after coronary artery bypass grafting, it did not improve in 15 patients $(17.6 \%)$ despite the presence of viable segments. The likelihood of the left ventricular ejection fraction recovery decreased proportionally with an increase in the left ventricular end-systolic volume. The nonimprovers had a higher left ventricular end-systolic volume $(164.2 \pm 22.4 \mathrm{~mL}$ vs $125.6 \pm 23.4 \mathrm{~mL}, P=.0001)$. In addition, the number of viable segments during the dobutamine stress echocardiography had a significant correlation with the ejection fraction increase after 6 months $(P<$ .0001). Patients with 6 viable segments showed a good outcome irrespective of their left ventricular end-systolic volume. In patients with fewer than 6 viable segments, left ventricular end-systolic volume was a major factor in the prognosis: Patients with left ventricular end-systolic volume of 145 or more had a poor left ventricular ejection fraction increase and vice versa.

Conclusion: The extent of left ventricular remodeling determines the rate of functional improvement after coronary artery bypass grafting. Patients with a high left ventric-

From the Day General Hospital, Tehran, Iran.

Received for publication July 4, 2007; revisions received Sept 3, 2007; accepted for publication Nov 1, 2007.

Address for reprints: Farideh Roshanali, MD, N0.1, 8th Floor, 15th Tower, Hormozan St, Ghods Shahrak, Tehran, 14466, Iran (E-mail: farideh_roshanali@yahoo. com).

J Thorac Cardiovasc Surg 2008;136:930-6 $0022-5223 / \$ 34.00$

Copyright $(2008$ by The American Association for Thoracic Surgery

doi:10.1016/j.jtcvs.2007.11.061 ular end-systolic volume and fewer than 6 viable segments have a lower likelihood of improvement.

$\mathrm{M}$ yocardial revascularization in patients with severe ischemic left ventricular (LV) dysfunction remains controversial because of concerns over operative mortality and morbidity and a lack of functional and survival benefits. ${ }^{1}$ Nevertheless, because medical treatment alone has a poor outlook, revascularization or cardiac transplantation may be considered for patients with ischemic cardiomyopathy. ${ }^{2,3}$

Operative mortality and early mortality (during a 30-day period after surgery) have been reported to be higher in patients with a low ejection fraction $(\mathrm{EF}),{ }^{4,5}$ and there seems to be a virtually linear increase in risk with the decreasing values of left ventricular ejection fraction $(\mathrm{LVEF}){ }^{5}$ The mortality rate at 1 year, however, is 

Abbreviations and Acronyms
$\mathrm{CABG}=$ coronary artery bypass grafting
DSE = dobutamine stress echocardiography
$\mathrm{EF} \quad=$ ejection fraction
$\mathrm{LV}=$ left ventricular
LVEDV = left ventricular end-diastolic volume
LVEF = left ventricular ejection fraction
LVESV = left ventricular end-systolic volume
LVESVI = left ventricular end-systolic volume index

significantly lower in patients with low EF who have undergone coronary artery bypass grafting $(\mathrm{CABG})$ than that of nonrevascularized patients with low $\mathrm{EF}^{4}$

CABG can be performed relatively safely in low EF cases, yielding an acceptable perioperative mortality risk and improvement in functional status, quality of life, and LV function (documented by a significant decrease in systolic LV dimensions and increase in EF). ${ }^{1,4,6,7}$

CABG is indicated in selected patients with ischemic cardiomyopathy. CABG may be offered to patients with impaired ventricular function; however, before opting for surgery, a rigorous patient selection and management requires the consideration of potentially reversible dysfunction (the presence of viable myocardium is necessary). ${ }^{1,8}$

It is important to recognize hibernating myocardium in patients with ischemic cardiomyopathy because symptoms resulting from chronic LV dysfunction may be due to a reversible ischemic process. ${ }^{9}$ It has emerged that approximately $50 \%$ of patients with ischemic cardiomyopathy have a substantial amount of hibernating myocardium. ${ }^{10,11}$

Myocardial contractility in viable myocardium can be reversible after revascularization. In patients with a considerable amount of viable myocardium, the failure to recover may be related to an increased LV volume in the wake of extensive ventricular remodeling. ${ }^{10,12,13}$

In this study, we investigated the effects of some preoperative factors, left ventricular end-systolic volume (LVESV), and the number of viable segments on LV function after revascularization in patients with ischemic cardiomyopathy. In addition, to facilitate the decision-making process for the suitable surgical procedure, we looked into the interaction of these factors to exactly predict which patients would show LV function improvement after CABG alone and which patients would not and, therefore, might need an additional procedure.

\section{Patients and Methods}

\section{Study Population and Protocol}

Between May of 2003 and April of 2004, 85 patients (63 men and 22 women) with a mean age of $59.5 \pm 13.8$ years (range: $30-81$ years) were studied. All subjects had symptomatic ischemic heart disease with a severely depressed LVEF $(\leq 35 \%)$ due to chronic coronary artery disease, which was confirmed by angiography. The patients underwent $\mathrm{CABG}$ under cardiopulmonary bypass according to their clinical status and coronary angiography.

Patients with primary cardiomyopathy, significant valvular heart disease (including ischemic mitral regurgitation), concomitant valve surgery, and LV aneurysm requiring repair were excluded from this study.

The study protocol was as follows: Regional wall-motion abnormality, LV volumes, and myocardial viability of the subjects were evaluated once pre-CABG via stress echocardiography and then 6 months afterward by echocardiography. Ethical approval was granted by the hospital's ethics committee, and written informed consent for inclusion in the study was obtained from the patients.

\section{Echocardiography}

All patients underwent preoperative dobutamine stress echocardiography (DSE) within 24 hours before CABG and postoperative transthoracic echocardiography 6 months after surgery.

The echocardiograms were obtained with a commercially available ultrasound machine (GE Vivid 7, Horten, Norway) with a 2.0 to 3.0-MHz phased-array transducer. The images were acquired from standard parasternal long- and short-axis planes, and from apical 4- and 2-chamber planes (in cine-loop format). All standard images were recorded on both optical disks and videotapes.

Left ventricular end-diastolic volume (LVEDV), LVESV, and LVEF were determined off-line by the cross-sectional biplane disk method using a modified Simpson's rule. The endocardial borders of the 2- and 4-chamber apical views were digitally traced at end diastole and end systole. An increased LVEF of 5\% or more after CABG was considered clinically significant, as described previously. ${ }^{10}$

\section{Assessment of Myocardial Viability}

Resting transthoracic echocardiography was followed by a low-dose DSE study. The dobutamine infusion was commenced with $2.5 \mu \mathrm{g} /$ $\mathrm{kg} / \mathrm{min}$, which was subsequently increased to $5,7.5$, and $10 \mu \mathrm{g} / \mathrm{kg}$ of body weight per minute in 3-minute stages. At each stage, the blood pressure and heart rate were measured. The end points of the test were significant ventricular and supraventricular arrhythmia, significant bradyarrhythmia, increased blood pressure of more than 240/ $120 \mathrm{~mm} \mathrm{Hg}$, decrease of $20 \mathrm{~mm} \mathrm{Hg}$ in the systolic blood pressure compared with the baseline value, new wall-motion abnormality, ST depression of $2 \mathrm{~mm}$ or more, and severe angina.

The left ventricle was divided according to the recommendations of the American Society of Echocardiography, and a 16-segment model was used. ${ }^{14}$ The images were displayed side by side in a quad-screen format at any stage. Regional wall motion and systolic wall thickening were scored using a 5-point grading scale: $1=$ normal; $2=$ mildly hypokinetic; $3=$ severely hypokinetic; $4=$ akinetic; and $5=$ dyskinetic. Segments with severe hypokinesia, akinesia, or dyskinesia were considered abnormal and evaluated for viability. Segments with improvement in wall-motion response were considered viable, and dysfunctional segments without change in contractility were considered nonviable.

\section{Statistical Analysis}

Data analyses were performed using the Statistical Package for the Social Sciences version 11.0 statistical analysis software (SPSS Inc, Chicago, Ill). Data were presented as mean \pm standard deviation for continuous variables and as numbers $(\%)$ for categoric 
variables. A Pearson's chi-square test was used to assess the categoric comparisons between the groups, and the Student $t$ test or analysis of variance was used for continuous measurements. Pearson's correlation coefficient was applied to show the correlation between the 2 continuous variables.

Linear regression enabled us to assess the independence of the factors related to the EF increase after 6 months. All independent variables were entered into the model, and the variables with a $P$ value greater than .1 were exited. For the model's goodness of fit, the $\mathrm{R}^{2}$ was presented.

The patients with considerable amounts of myocardial viability were divided into 2 groups: those with and without improvement in the LV function after revascularization (an LVEF increase of $\geq$ $5 \%$ and an LVEF increase of $<5 \%$, respectively).

\section{Results}

\section{Patient Characteristics}

The clinical and echocardiographic characteristics of the study population are presented in Table 1. Eighty-five patients, consisting of 63 men $(74.1 \%)$ and 22 women (25.9\%) with a mean age of $59.5 \pm 13.8$ years, with ischemic cardiomyopathy and substantial viability were studied. All patients had heart failure symptoms with an average New York Heart Association functional class of $2.8 \pm 0.7$. The range of preoperative $\mathrm{EF}$ was $20 \%$ to $35 \%$ with a mean $\mathrm{EF}$ of $27.2 \% \pm 5.3 \%$. The mean LVESV was $132.4 \pm 27.4$. The number of stenosed arteries was $2.6 \pm 0.6$. Perioperative myocardial infarction, documented with cardiac enzyme increasing, was not detected in the patients. An inotropic infusion was used in 31 patients $(36.5 \%)$ postoperatively. Eleven patients $(12.9 \%)$ required intra-aortic balloon pumps. Ventricular assist devices were not used in the patients.

\section{Contractility and Viability Status}

A total of 1360 segments were evaluated with 2-dimensional echocardiography. There were 565 segments $(41.5 \%)$ with normal contractility and 795 segments $(58.5 \%)$ with abnormal contractility, that is, severe hypokinesia, akinesia, and dyskinesia.

\section{TABLE 1. Patient characteristics}

\begin{tabular}{lc}
\hline \multicolumn{1}{c}{ Clinical features } \\
\hline Age (y) & $59.5 \pm 13.8$ \\
Male & $63(74.1 \%)$ \\
Previous infarction & $73(85.9 \%)$ \\
No. of stenosed arteries & $2.6 \pm 0.6$ \\
NYHA functional class & $2.8 \pm 0.7$ \\
Baseline LVEF & $27.2 \% \pm 5.3 \%$ \\
No. of normal segments & $6.6 \pm 1.4$ \\
LVESV (mL) & $132.4 \pm 27.4$ \\
\hline
\end{tabular}

NYHA, New York Heart Association; LVEF, left ventricular ejection fraction; $L V E S V$, left ventricular end-systolic volume. Data are presented as mean \pm standard deviation or as numbers (\%).
DSE was only performed before CABG. The dysfunctional segments were composed of 426 (53.6\%) viable segments (because of functional improvement during DSE) and $369(46.4 \%)$ nonviable segments. After the dobutamine infusion, the mean EF increase was $12.1 \% \pm 3.6 \%$, with a minimum increase of $5 \%$. Although post-CABG recovery in the $\mathrm{LV}$ function (increase of $\geq 5 \%$ in $\mathrm{EF}$ ) was expected in those with substantial viability, LVEF improved in only 70 patients $(82.4 \%)$. In 15 patients $(17.6 \%)$, LVEF failed to improve despite the presence of considerable amounts of viability. In the total study population, the average postoperative improvement in LVEF was $9.9 \% \pm 5.9 \%$.

Pearson's correlation coefficient for the EF increase after 6 months and the number of the viable segments during the DSE test was $\mathrm{R}=0.51(P<.0001)$; the same coefficient for the correlation between the EF increase after 6 months and the number of the recovered segments after 6 months was $\mathrm{R}=0.84(P<.0001)$.

\section{Comparison Between Left Ventricular Function Improvers and Nonimprovers}

Table 2 shows a comparison between the LV function improvers and nonimprovers. The clinical characteristics and echocardiographic data were comparable between the 2 groups (LVEF increase $\geq 5 \%$ and LVEF increase $<5 \%$ ). We obtained data independently of LVESV, which was different in the 2 groups. The nonimprovers had a significantly larger LVESV than the improvers $(164.2 \pm 22.4 \mathrm{~mL}$ vs $125.6 \pm 23.4 \mathrm{~mL}$, respectively; $P=.0001)$. The average numbers of stenosed coronary arteries in the LV function improvers and nonimprovers were $2.5 \pm 0.6$ and $2.8 \pm 0.4$, respectively $(P=.047)$.

In the univariate analysis, the number of stenosed arteries, baseline LVEF, number of normal segments at rest, number

TABLE 2. Comparison between patients with substantial viability with or without improvement in left ventricular function after revascularization

\begin{tabular}{lccr}
\hline \multicolumn{1}{c}{ Clinical features } & LVEF improvers & LVEF nonimprovers & \\
\hline Age (y) & $59.2 \pm 15.1$ & $60.5 \pm 2.9$ & .510 \\
Male gender & $53(75.7 \%)$ & $10(66.7 \%)$ & .468 \\
Previous infarction & $60(85.7 \%)$ & $13(92.9 \%)$ & .470 \\
No. of stenosed arteries & $2.53 \pm 0.63$ & $2.80 \pm 0.41$ & .047 \\
No. of grafts & $3.69 \pm 1.12$ & $3.73 \pm 1.10$ & .882 \\
NYHA functional class & $2.80 \pm 0.73$ & $2.80 \pm 0.68$ & 1.000 \\
Baseline LVEF & $27.9 \% \pm 5.5 \%$ & $23.9 \% \pm 2.7 \%$ & .000 \\
No. of normal segments & $6.8 \pm 1.5$ & $6.0 \pm 0.0$ & .000 \\
LVESV (mL) & $125.6 \pm 23.4$ & $164.2 \pm 22.4$ & .000 \\
No. of viable segments & $5.2 \pm 0.8$ & $4.3 \pm 0.5$ & .000 \\
$\quad$ during DSE & & & \\
\hline
\end{tabular}

NYHA, New York Heart Association; LVEF, left ventricular ejection fraction; $\angle V E S V$, left ventricular end-systolic volume; DSE, dobutamine stress echocardiography. 
TABLE 3. Variables with significant correlations with the increase in ejection fraction

\begin{tabular}{|c|c|c|c|c|c|}
\hline & \multicolumn{2}{|c|}{ Unstandardized coefficients } & \multirow{2}{*}{$\frac{\text { Standardized coefficients }}{\text { Beta }}$} & \multirow[b]{2}{*}{$t$} & \multirow[b]{2}{*}{$\boldsymbol{P}$} \\
\hline & B & SE & & & \\
\hline (Constant) & 84.022 & 11.295 & & 7.439 & .000 \\
\hline Age & -0.129 & 0.025 & -0.300 & -5.212 & .000 \\
\hline LVESV & -0.622 & 0.086 & -2.887 & -7.235 & .000 \\
\hline No. of viable segments during DSE & -10.953 & 2.343 & -1.580 & -4.675 & .000 \\
\hline No. of viable segments during DSE * LVESV & 0.107 & 0.018 & 3.043 & 6.110 & .000 \\
\hline
\end{tabular}

$S E$, Standard error; LVESV, left ventricular end-systolic volume; DSE, dobutamine stress echocardiography. Number of viable segments during DSE * LVESV. Interaction between the number of viable segments during DSE and LVESV. $\mathrm{R}^{2}=0.75$.

of the viable segments during DSE, and LVESV were different between the 2 groups. In the regression model, however, only age, LVESV, the number of viable segments during DSE, and the interaction between the 2 variables of LVESV and the number of viable segments during DSE had a significant correlation with the increase in EF (Table 3).

As we mentioned, there was an interaction between LVESV and the number of viable segments during DSE; we assessed the mean of the EF increase at different numbers of viable segments during the DSE study in 2 levels of LVESV $(<145$ and $\geq 145 \mathrm{~mL}$ ) (Figure 1). The existence of 6 or more viable segments discovered during DSE was predictive of a good surgical outcome despite a large LVESV. On the other hand, when there were fewer than 6 viable segments detected via the DSE study, LVESV played a major role in the outcome prediction: Patients with LVESV less than $145 \mathrm{~mL}$ improved, whereas patients with LVESV of $145 \mathrm{~mL}$ or more did not.

\section{Discussion}

Surgical revascularization in patients with a severe impairment of the LV function yields more favorable outcomes if patient selection and management are performed thoroughly.

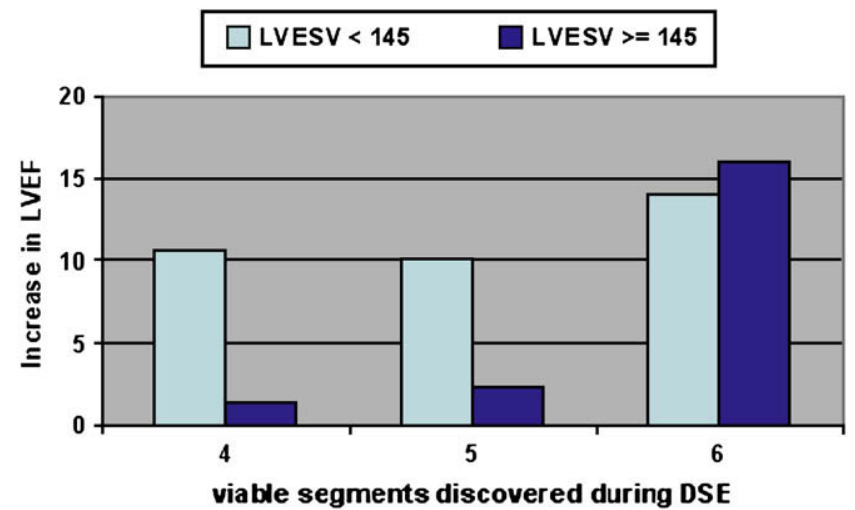

Figure 1. The amount of the LVEF increase based on the number of viable segments during preoperative DSE in different LVESV groups. LVESV, Left ventricular end-systolic volume; DSE, dobutamine stress echocardiography; LVEF, left ventricular ejection fraction.
It has been posited that an accurate prediction of the postoperative functional recovery in patients with ischemic cardiomyopathy and a mixture of viable and nonviable tissues requires the consideration of the number of both viable and nonviable (scar) segments. ${ }^{15}$

After revascularization, improvement in LV function and prognosis is expected in patients with considerable amounts of myocardial viability. Nonetheless, many patients with substantial viability in their dysfunctional myocardium do not exhibit such functional improvement, which could be explained by the increased LV volume after extensive ventricular remodeling. ${ }^{12,13}$

The present study evaluated 85 patients with substantial viability ( $\geq 4$ viable segments) once pre-CABG and then 6 months post-CABG. Despite substantial viability and expectation of improvement in the global function after surgery, the LV function failed to recover in $15(17.6 \%)$ of our subjects.

A subsequent comparison between the LVEF improvers and nonimprovers produced the following results: Baseline LVEF, number of stenosed arteries, number of normal segments before CABG, LVESV, and number of viable segments during DSE were significantly different between the 2 groups. With Pearson's correlation coefficient, however, only age, LVESV, and number of viable segments during DSE were found to have a significant correlation with the LVEF increase after CABG.

In patients undergoing $\mathrm{CABG}$, the operative mortality and morbidity are higher in patients with severe LV dysfunction and substantial viable myocardium than in those with a normal LV function. Although a low EF is the major risk factor for operative mortality, EF alone may not necessarily be an accurate predictor of operative mortality. ${ }^{16}$ Kawachi and colleagues ${ }^{16}$ assessed the correlations between a large left ventricle with LV dysfunction and operative mortality in 106 patients with an EF of $40 \%$ or less. In their study, the hospital mortality in the patients with an EF less than $40 \%$ and an LVESV of $140 \mathrm{~mL} / \mathrm{m}^{2}$ or more was $50 \%$; and the hospital mortality in the patients with an LVEDV of $200 \mathrm{~mL} / \mathrm{m}^{2}$ or more was $67 \%$. The authors did not evaluate the LVEF improvement after revascularization. They concluded that patients with a low EF and an elevated LVESV or LVEDV were at increased risk for hospital death after CABG. ${ }^{16}$ 
In a more recent study, Trehan and colleagues ${ }^{17}$ studied 176 patients with an LVEF of less than $30 \%$ who underwent isolated CABG. Having evaluated predictors of survival and early outcome after coronary artery surgery, they posited that the left ventricular end-systolic volume index (LVESVI) was the only independent parameter of the LV function predictive of survival. They also did not assess the LVEF improvement after revascularization.

Yamaguchi and colleagues ${ }^{12}$ demonstrated that the mean EF improved significantly after CABG in patients with a preoperative LVESVI of less than $100 \mathrm{~mL} / \mathrm{m}^{2}$ despite the presence of a global LVEF of less than $30 \%$. They determined the preoperative and postoperative EF, LVESVI, and LVEDV index by using biplane left cineventriculography. However, they chose not to assess viability. Another study demonstrated that the improvement in LVEF was more prominent in patients with a preoperative LV end-diastolic diameter of less than $70 \mathrm{~mm} .^{8}$

Rizzello and colleagues ${ }^{18}$ evaluated the probability of the LV functional recovery after CABG via resting 2-dimensional echocardiography; they did not make use of DSE in their study. The cutoff value of an end-diastolic volume index of $90 \mathrm{~mL}$ or more accurately identified patients who would virtually never recover. It was concluded that patients with ischemic cardiomyopathy and severe LV enlargement were unlikely to exhibit improvement in LVEF after revascularization. Conversely, patients with a relatively preserved LV size had a higher likelihood of functional recovery.

Bax and colleagues ${ }^{19}$ evaluated recovery in the LV function after $\mathrm{CABG}$ and long-term prognosis in patients with extensive LV remodeling. LVESV was the only parameter that was significantly different between the groups $(109 \pm 46 \mathrm{~mL}$ for the improvers vs $141 \pm 31 \mathrm{~mL}$ for the nonimprovers). The change in LVEF after revascularization was linearly related to the baseline LVESV: The higher the LVESV, the lower the likelihood of improvement in LVEF after revascularization. Viability was assessed by means of metabolic imaging with F18-fluorodeoxyglucose and single photon emission computed tomography. During a 3-year follow-up period, the highest event rate $(67 \%)$ was observed in patients without viable myocardium and a large LV size, whereas the lowest event rate $(5 \%)$ was observed in patients with viable myocardium and a small LV size. The authors concluded that extensive LV remodeling not only prohibited improvement in LVEF after revascularization but also affected long-term prognosis negatively despite considerable viability.

Schinkel and colleagues ${ }^{10}$ studied 118 patients (61 patients had substantial viability) with an average LVEF of $29 \%$ due to chronic coronary artery disease. The subjects underwent revascularization, and DSE and radionuclide ventriculography were used for the assessment of viability and determination of LVEF, respectively. LVEF did not improve in $33 \%$ of 61 patients despite substantial viability. The nonimprovers had a considerably larger LVESV $(153 \pm 41 \mathrm{~mL}$ vs
$133 \pm 46 \mathrm{~mL}$ ). The likelihood of the recovery of the global function decreased proportionally with the increase in LVESV. The researchers demonstrated that an LVESV of $140 \mathrm{~mL}$ or more had the highest sensitivity and specificity for the prediction of the absence of the global recovery.

It can be concluded that the existence of viability in the dysfunctional tissue, albeit essential, is not sufficient for post-CABG LVEF improvement and that some other factors must exert a significant influence. Usually a value of 4 or more viable segments is advised as a cutoff value for the prediction of the LVEF improvement. ${ }^{20,21}$ The presence of this level of viability ( $25 \%$ of LV mass) predicts patients who may benefit from revascularization. ${ }^{20,21}$ Because revascularization alone cannot improve LVEF in patients with an increased LVESV even if there is substantial viability (ie, $\geq$ 4 viable segments), not only the presence of myocardial viability but also the cardiac remodeling and enlargement of the left ventricle should be taken into consideration. This may be helpful in case selection before CABG: Patients with an increased LVESV as a result of extensive cardiac remodeling may require more viable segments to ensure a good postCABG prognosis. On the other hand, in the present study a significant correlation was found between the number of viable segments during preoperative DSE and the EF increase after CABG, and patients with 6 or more viable segments showed an encouraging post-CABG outcome. Our findings in this study agree with those of previous studies. ${ }^{22}$

In addition to some known predicting factors (eg, LVESV and LVEDV) reflecting the severity of cardiac remodeling, some other factors (eg, number of viable segments during preoperative DSE) should be drawn on to predict who may benefit from revascularization. Considering both the severity of cardiac remodeling and the number of viable segments during preoperative DSE must be superior to taking into account either alone. Although the above-mentioned studies have accurately described the cutoff values for LVESV and the amount of viability, to our knowledge none have evaluated the interaction between these predicting factors. We, however, used the 2 predicting factors of LVESV and the number of viable segments to make a prognosis in patients with ischemic cardiomyopathy scheduled for CABG. As depicted in Figure 1, the postoperative functional recovery was encouraging in patients who had 6 viable segments. In the other patients with fewer than 6 viable segments, LVESV played a key role. Moreover, the patients with an LVESV of less than $145 \mathrm{~mL}$ showed an acceptable outcome, whereas patients with an LVESV of $145 \mathrm{~mL}$ or more did not improve.

An increased LVESV inhibits the functional recovery after CABG in patients without a large mass of viable segments (6 viable segments). In these patients with an enlarged left ventricle, $\mathrm{CABG}$ should be combined with $\mathrm{LV}$ restoration to improve outcomes compared with CABG alone even in the absence of aneurysm. LV restoration affords significant improvement in $\mathrm{EF}$ in comparison with $\mathrm{CABG}$ alone without 
added mortality. Surgical reduction of akinetic and dyskinetic segments may reduce wall stress and break the vicious cycle of cardiac remodeling and consequently improve the LV geometry and function in selected patients. ${ }^{23,24}$ More important, LV restoration reduces late morbidity and mortality compared with CABG alone in patients with large ventricles. ${ }^{25}$ Future studies are necessary to identify how to approach the patients with substantial dysfunctional but viable myocardium accompanied by extensive LV enlargement.

\section{Study Limitations}

All of the significantly stenosed vessels were subjected to complete revascularization. Post-CABG follow-up coronary angiography was not performed; therefore, it can be argued that the presence of graft occlusion is likely to have precluded improvement in the LV function. In addition, the drugs used for the treatment of heart failure and ischemic heart disease (eg, beta-blockers and angiotensin-converting enzyme inhibitors) may have affected the LV function.

Our results were obtained over a 6-month period. Needless to say, a longer period of time may have yielded better results in LV function improvement. The small sample size and the resultant limitations in the multivariate statistical analysis in this study call for further research with a more extended scope.

\section{Conclusions}

In patients with ischemic cardiomyopathy accompanied by fewer than 6 viable segments during a preoperative DSE study, the extent of LV remodeling and dilatation is important in making an accurate prediction of the LV function improvement after coronary revascularization. An increased LVESV in the wake of extensive LV remodeling reduces the likelihood of improvement in the global function; therefore, another surgical procedure may be required to improve the LV function in this group of patients. On the other hand, in patients with a large mass of viable myocardium (ie, 6 viable segments) $\mathrm{CABG}$ has a favorable outcome irrespective of LVESV.

\section{References}

1. Bouchart F, Tabley A, Litzler PY, Haas-Hubscher C, Bessou JP, Soyer R. Myocardial revascularization in patients with severe ischemic left ventricular dysfunction. Long term follow-up in 141 patients. Eur J Cardiothorac Surg. 2001;20:1157-62.

2. Lytle BW. The role of coronary revascularization in the treatment of ischemic cardiomyopathy. Ann Thorac Surg. 2003;75(Suppl):S2-5.

3. Baker DW, Jones R, Hodges J, Massie BM, Konstam MA, Rose EA. Management of heart failure. III. The role of revascularization in the treatment of patients with moderate or severe left ventricular systolic dysfunction. JAMA. 1994;272:1528-34.

4. Appoo J, Norris C, Merali S, Graham MM, Koshal A, Knudtson ML, et al. Long-term outcome of isolated coronary artery bypass surgery in patients with severe left ventricular dysfunction. Circulation. 2004; 110(Suppl 1):II13-7.

5. Risum O, Nitter-Hauge S, Abdelnoor M, Levorstad K, Arafa O, Svennevig JL. Mortality and morbidity after coronary artery bypass surgery related to pre-operative left ventricular ejection fraction: a follow-up study. Eur Heart J. 1996;17:874-9.

6. De Carlo M, Milano A, Borzoni G, Pratali S, Barzaghi C, Tartarini G, et al. Predicting outcome after myocardial revascularization in patients with left ventricular dysfunction. Cardiovasc Surg. 1998;6:58-66.

7. Carr JA, Haithcock BE, Paone G, Bernabei AF, Silverman NA. Longterm outcome after coronary artery bypass grafting in patients with severe left ventricular dysfunction. Ann Thorac Surg. 2002;74:1531-6.

8. Nemec P, Bedanova H, Necas J, Meluzin J, Stetka F, Pokorny P, et al. Coronary artery bypass grafting in patients with left ventricular ejection fraction of $30 \%$ or less. Bratisl Lek Listy. 2001;102:15-21.

9. Schinkel AF, Bax JJ, Sozzi FB, Boersma E, Valkema R, Elhendy A, et al. Prevalence of myocardial viability assessed by single photon emission computed tomography in patients with chronic ischaemic left ventricular dysfunction. Heart. 2002;88:125-30.

10. Schinkel AF, Poldermans D, Rizzello V, Vanoverschelde JL, Elhendy A, Boersma E, et al. Why do patients with ischemic cardiomyopathy and a substantial amount of viable myocardium not always recover in function after revascularization? J Thorac Cardiovasc Surg. 2004; 127:385-90.

11. Schinkel AF, Bax JJ, Boersma E, Elhendy A, Roelandt JR, Poldermans D. How many patients with ischemic cardiomyopathy exhibit viable myocardium? Am J Cardiol. 2001;88:561-4.

12. Yamaguchi A, Ino T, Adachi H, Mizuhara A, Murata S, Kamio H. Left ventricular end-systolic volume index in patients with ischemic cardiomyopathy predicts postoperative ventricular function. Ann Thorac Surg. 1995;60:1059-62.

13. Louie HW, Laks H, Milgalter E, Drinkwater DC Jr, Hamilton MA, Brunken RC, et al. Ischemic cardiomyopathy. Criteria for coronary revascularization and cardiac transplantation. Circulation. 1991; 84(Suppl):III290-5.

14. Elefteriades JA, Morales DL, Gradel C, Tollis G Jr, Levi E, Zaret BL. Results of coronary artery bypass grafting by a single surgeon in patients with left ventricular ejection fractions $<$ or $=30 \%$. Am J Cardiol. 1997 ; 79:1573-8.

15. Rizzello V, Schinkel AF, Bax JJ, Boersma E, Bountioukos M, Vourvouri EC, et al. Individual prediction of functional recovery after coronary revascularization in patients with ischemic cardiomyopathy: the scar-to-biphasic model. Am J Cardiol. 2003;91:1406-9.

16. Kawachi K, Kitamura S, Hasegawa J, Kawata T, Kobayashi S, Mizuguchi $\mathrm{K}$, et al. Increased risk of coronary artery bypass grafting for left ventricular dysfunction with dilated left ventricle. J Cardiovasc Surg (Torino). 1997;38:501-5.

17. Trehan N, Khanna SN, Mishra Y, Kohli V, Mehta Y, Mishra M, et al. Predictors of early outcome after coronary artery surgery in patients with severe left ventricular dysfunction. J Card Surg. 2003;18:101-6.

18. Rizzello V, Bax JJ, Schinkel AF, Boersma E, Bountioukos M, Vourvouri EC, et al. Does resting two-dimensional echocardiography identify patients with ischemic cardiomyopathy and low likelihood of functional recovery after coronary revascularization? Coron Artery Dis. 2004;15:269-75.

19. Bax JJ, Schinkel AF, Boersma E, Elhendy A, Rizzello V, Maat A, et al. Extensive left ventricular remodeling does not allow viable myocardium to improve in left ventricular ejection fraction after revascularization and is associated with worse long-term prognosis. Circulation. 2004; 110(Suppl 1):II18-22.

20. Bax JJ, Poldermans D, Elhendy A, Cornel JH, Boersma E, Rambaldi R, et al. Improvement of left ventricular ejection fraction, heart failure symptoms and prognosis after revascularization in patients with chronic coronary artery disease and viable myocardium detected by dobutamine stress echocardiography. J Am Coll Cardiol. 1999;34:163-9.

21. Nagueh SF, Vaduganathan P, Ali N, Blaustein A, Verani MS, Winters WL, et al. Identification of hibernating myocardium: comparative accuracy of myocardial contrast echocardiography, rest-redistribution thallium-201 tomography and dobutamine echocardiography. J Am Coll Cardiol. 1997;29:985-93.

22. Meluzín J, Cerný J, Groch L, Frélich M, Stetka F, Spinarová L, et al. Prognostic importance of the quantification of myocardial viability in revascularized patients with coronary artery disease and moderate-to-sever left ventricular dysfunction. Int J Cardiol. 2003;90:23-31. 
23. Dor V, Sabatier M, Di Donato M, Montiglio F, Toso A, Maioli M. Efficacy of endoventricular patch plasty in large postinfarction akinetic scar and severe left ventricular dysfunction: comparison with a series of large dyskinetic scars. J Thorac Cardiovasc Surg. 1998;116:50-9.

24. Athanasuleas CL, Stanley AW Jr, Buckberg GD, Dor V, DiDonato M, Blackstone EH. Surgical anterior ventricular endocardial restoration (SAVER) in the dilated remodeled ventricle after anterior myocardial infarction. RESTORE group. Reconstructive Endoventricular Surgery, returning Torsion Original Radius Elliptical Shape to the LV. J Am Coll Cardiol. 2001;37:1199-209.

25. Maxey TS, Reece TB, Ellman PI, Butler PD, Kern JA, Tribble CG, et al. Coronary artery bypass with ventricular restoration is superior to coronary artery bypass alone in patients with ischemic cardiomyopathy. J Thorac Cardiovasc Surg. 2004;127:428-34. 